\title{
Length Tension Function of Puborectalis Muscle: Implications for the Treatment of Fecal Incontinence and Pelvic Floor Disorders
}

\author{
Ravinder K Mittal, ${ }^{1 *}$ Geoff Sheean, ${ }^{2}$ Bikram S Padda ${ }^{1}$ and Mahadevan R Rajasekaran ${ }^{1}$ \\ Pelvic Floor Function and Diseases Group, ${ }^{1}$ Division of Gastroenterology and ${ }^{2}$ Department of Neurology, University of California, San Diego, \\ CA, USA
}

\begin{abstract}
Background/Aims
External anal sphincter (EAS) and puborectalis muscle (PRM) play important role in anal continence function. Based on length-tension measurement, we recently reported that the human EAS muscle operates at short sarcomere length under physiological conditions. Goal of our study was to determine if PRM also operates at the short sarcomere length.

\section{Methods}

Length-tension relationship of the PRM muscle was studied in vivo in 10 healthy nullipara women. Length was altered by vaginal distension using custom-designed probes of 5, 10, 15, 20, 25 and $30 \mathrm{~mm}$ diameters as well as by distending a polyethylene bag with different volumes of water. Probes were equipped with a reverse perfuse sleeve sensor to measure vaginal pressure (surrogate of PRM tension). PRM electromyogram (EMG) was recorded using wire electrodes. Three-dimensional ultrasound images were obtained to determine effect of vaginal distension on PRM length.
\end{abstract}

\section{Results}

Ultrasound images demonstrate distension volume dependent increase in PRM length. Rest and squeeze pressures of vaginal bag increased with the increase in bag volume. Similarly, the change in vaginal pressure, which represents the PRM contraction increased with the increase in the probe size. Increase in probe size was not associated with an increase in EMG activity (a marker of neural drive) of the PRM.

\section{Conclusions}

Probe size dependent increase in PRM contraction pressure, in the presence of constant EMG (neural input) proves that the human PRM operates at short sarcomere length. Surgically adjusting the PRM length may represent a novel strategy to improve treat anal continence and possibly other pelvic floor disorders.

\section{(J Neurogastroenterol Motil 2014;20:539-546)}

\section{Key Words}

Anal continence; Anal sphincter; Function; Muscles; Puborectalis muscle

Received: March 19, 2014 Revised: April 25, 2014 Accepted: April 26, 2014

(c) This is an Open Access article distributed under the terms of the Creative Commons Attribution Non-Commercial License (http://creativecommons. org/licenses/by-nc/3.0) which permits unrestricted non-commercial use, distribution, and reproduction in any medium, provided the original work is properly cited.

*Correspondence: Ravinder K Mittal, MD

Department of Medicine, University of California, San Diego, CA 92161, USA

Tel: +1-858-552-7556, Fax: +1-858-552-7436, E-mail: rmittal@ucsd.edu

Financial support: This study was supported by Veterans Administration MERIT Grant, NIH Grant (DK091348) and a Grant from the American Society of Colon and Rectal Surgeons.

Conflicts of interest: None.

Author contributions: Ravinder K Mittal, concept, experimental design, interpretation of data, grant funding, data analysis and manuscript writing; Geoff Sheean, study design, electromyographic and functional studies; Bikram S Padda, data acquisition and analysis; Mahadevan R Rajasekaran, data analysis, interpretation of data and manuscript writing.

ORCID: Ravinder K mittal, http://orcid.org/0000-0001-5228-9617. 


\section{Introduction}

Internal anal sphincter (IAS), external anal sphincter (EAS) and puborectalis muscle (PRM) are important components of the anal sphincter complex ${ }^{1-3}$ and together they contribute to the anal sphincter pressure and anal continence function. It is generally believed that anal canal pressure at rest is related to the tonic contraction of IAS and the increase in pressure with voluntary squeeze is due to the EAS. Recent studies suggest that voluntary contractions of PRM and EAS increase pressure in the proximal and distal halves of anal canal, respectively. ${ }^{4,5}$ In addition to anal closure, PRM a "U" shaped pelvic floor muscle causes closure of vagina and urethra as well. ${ }^{6-8}$

Skeletal muscles, such as EAS and PRM, contract in response to central input from the cortical center of brain. Neural impulses from cortical center impinge on the anterior horn cells of the spinal cord, which communicate to skeletal muscle through peripheral nerves, pudendal nerve and sacral nerve roots (S3 and S4) in the case of PRM and pelvic floor muscles. ${ }^{9}$ Under physiological conditions, central input (neural drive to the muscle) and muscle length/sarcomere length are the key determinants of force/tension generated by the skeletal muscles. ${ }^{10}$

Central drive or neural input to a muscle is measured by electromyographic (EMG) activity of muscle and increase in vaginal pressure with voluntary squeeze is a surrogate marker of the force/stress generated by the PRM. ${ }^{6,9}$ Recent studies from our laboratory show that EAS muscle operates at a short sarcomere length in rabbits and humans, ${ }^{11-14}$ i.e., increasing its in vivo length increase its contraction pressure. Whether the same is true for PRM in humans is not known and was the purpose of our present investigation. A clear understanding of the above phe- nomenon would provide rationale for the possibility that surgical adjustment of the PRM length may be used to treat anal continence and possibly other pelvic floor disorders.

\section{Materials and Methods}

The UCSD Institutional Review Board (Project No. 071326) approved our study protocol and each subject signed an informed consent prior to participation in the study. Ten nullipara women (mean age, 33; range, 23-55; 8 caucasians and 2 asians) participated in the study protocol. Each subject completed medical history, urinary and fecal incontinence-scoring questionnaires to confirm the absence of urinary and fecal incontinence symptoms. Subjects were instructed to contract pelvic floor muscles by the prompt: "squeeze as if you were trying to prevent bowel movement or hold stream of urine." Subjects were instructed to squeeze for 10 seconds and then relax for 30 seconds in between squeezes to minimize pelvic floor muscle fatigue.

Vaginal pressure was measured using manometry catheter equipped with a $6 \mathrm{~cm}$ long reverse water-perfuse sleeve sensor. ${ }^{15,16}$ We developed a novel probe system to perform vaginal distension and at the same time measure vaginal pressure. Probe system consists of placing a $5 \mathrm{~mm}$ diameter sleeve sensor in the custom built probe holders of 10, 15, 20, 25 and $30 \mathrm{~mm}$ diameters. The probe holders were made of noncompliant material (propylene) and contained a groove for the placement of sleeve-sensor manometry catheter. Sleeve sensor was placed in the holders in such a fashion that the pressure-sensing surface of the sleeve faced outwards (Fig. 1). Sleeve sensor along with the holder was placed in vagina with the pressure-sensing surface of sleeve facing anterior midline direction. All pressures were measured in reference to atmospheric pressure. Rest pressure was determined as the highest
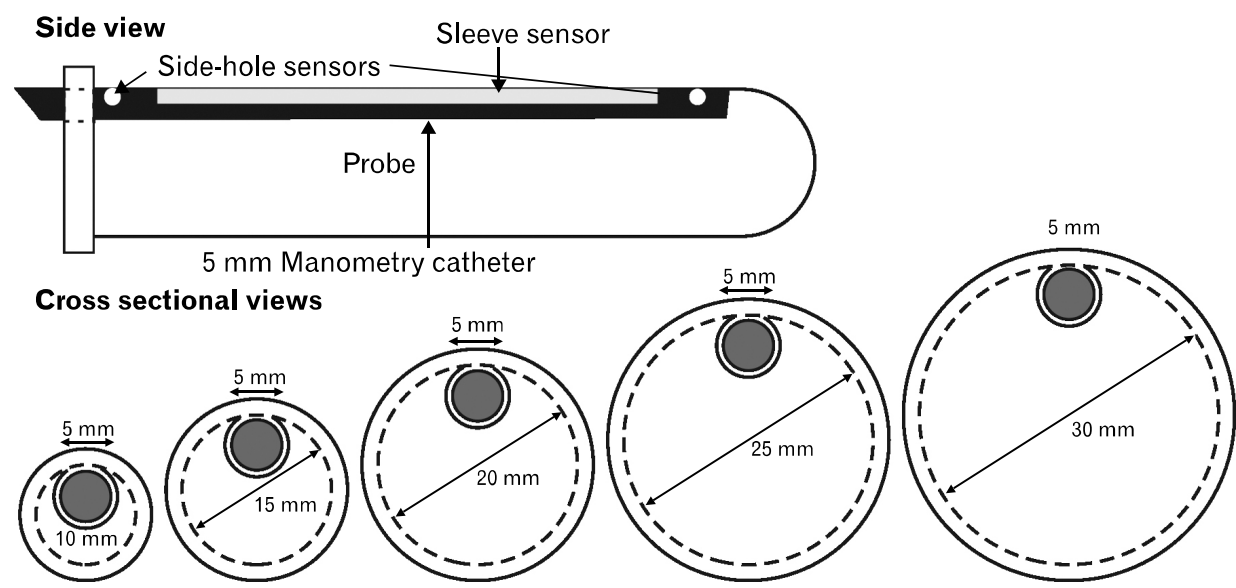

Figure 1. Schematics of the manometry catheter and probe holder. Pressure is recorded using a $5 \mathrm{~mm}$ diameter manometry catheter which is placed inside probe holders of 10, 15, 20, 25 and $30 \mathrm{~mm}$ diameter. 
pressure recorded 5 seconds before the subject was asked to squeeze. Squeeze pressure was the peak pressure recorded during 10 -second squeeze period. "Delta pressure" is the difference between squeeze and rest pressures.

EMG activity of PRM was recorded using bipolar, wire hook electrodes (Viasys Healthcare, Madison, WI, USA) by Dr Sheean (neurologist) with considerable experience in these recording. Briefly, needle containing wires was inserted trans-cutaneously, $1-2 \mathrm{~cm}$ from the anal verge, at 9 o'clock position to a depth of 3-3.5 cm from the skin surface. EMG recording was obtained during insertion to confirm electrical quiescence, as the needle traversed from the EAS into PRM, which is an important criterion for the passage of needle from EAS into PRM. Bandwidth of the recorded signal was between 20-500 Hz. Raw EMG signal was integrated and a moving time (every 200 millisecond) average signal was recorded continuously during the entire study period and quantified in arbitrary units. ${ }^{17}$ Rest and peak EMG activity was measured at the same time points as the rest and squeeze vaginal pressures. All signals (pressure and EMG) were recorded using a Medtronics Recorder (Medtronics, Minneapolis, MN, USA).

To determine the effect of vaginal distension on PRM length, a compliant polyethylene bag was placed in vagina and filled with various volumes of water. Part of these data were presented earlier in reference to characterization of vaginal high pressure zone. ${ }^{6}$ Characteristics of polyethylene bag were as follows: it was cylindrical in shape, $10 \mathrm{~cm}$ in length and $35 \mathrm{~mm}$ in maximal diameter when fully distended. A catheter with multiple side holes along its length was placed inside the bag for infusion of water to distend the bag and therefore the vagina. Ex vivo studies were conducted before placement of bag in the vagina to determine maximal volume of water to be used for the in vivo studies. Bag was connected to a pressure transducer and gradually filled with water using a hand-held syringe. Volume of water that caused first increase in bag pressure was the called maximal volume. All bag distensions for in vivo experiments were performed with less than the maximal volume. Bag was positioned in the vagina such that approximately $2 \mathrm{~cm}$ of it was located below the hymen. Bag was inflated with water volumes that ranged from 45-70 $\mathrm{mL}$ in 5-mL increments. At each bag volume, a 3D ultrasound (US) volume of pelvis was obtained by placing a 5-9 $\mathrm{MHz}$ transvaginal US transducer on the perineum (Philips HD11 system; Phillips Medical Systems, Bothell, WA, USA), as described previously. ${ }^{6,7}$ US volumes were captured at rest and during sustained pelvic floor contractions with subject in the lithotomy position. US volumes were viewed with Q-lab 4.2 software program (Phillips Medical Systems) and displayed in a standard, symmetric orientation for the study of 2D mid sagittal image of pelvis. PRM length, which is same as pelvic floor hiatus length was measured as the distance between 2 points on the US image, lower end of pubic symphysis and apex of the anorectal angle (Fig. 2).

\section{Statistical Methods}

Data are shown as means \pm SEM. A 2-way repeated measure ANOVA with post hoc Tukey's test (SPSS Inc, Chicago, IL, USA) was used to determine the effect of probe size (PRM length) on the vaginal pressure and EMG activity. Linear regression analysis was used to determine the correlation between pressure and anterior-posterior length of the pelvic floor hiatus (PRM length).

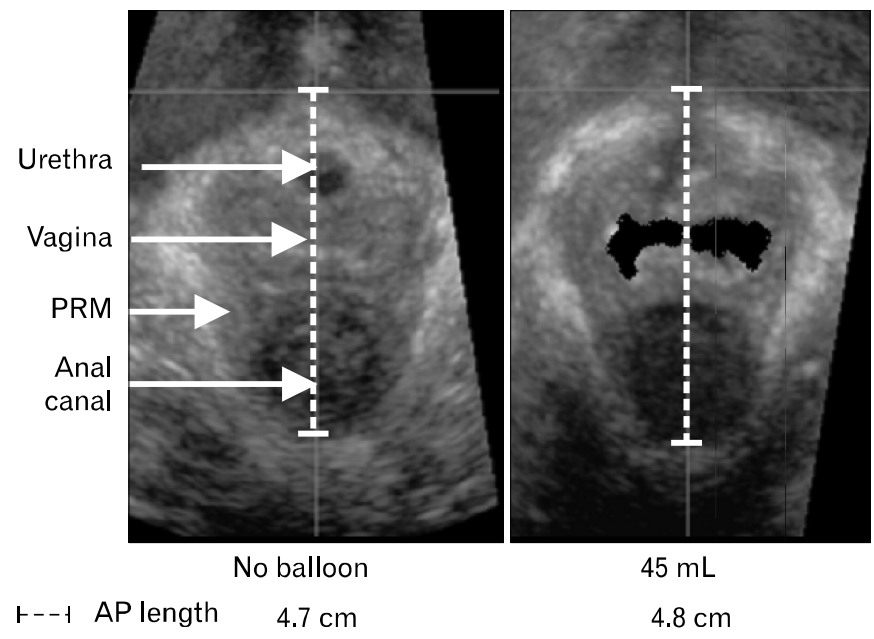

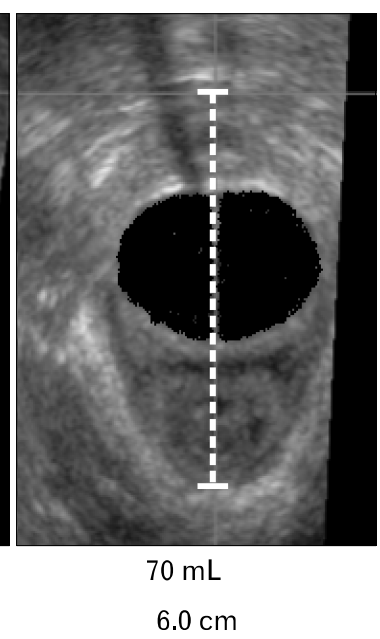

Figure 2. Transverse ultrasound (US) images of the pelvic floor hiatus. US images demonstrate vaginal distension dependent increase in the puborectalis muscle (PRM) length. AP, anterior posterior. 


\section{Results}

\section{Effect of Vaginal Distension on the Puborectalis Muscle Length and Bag Pressure}

Figure 2 shows transverse US images of the pelvic floor hiatus and Figure 3 summarizes anterior posterior (AP) length changes of PRM with vaginal distension. AP length of the PRM decreased significantly during pelvic floor contraction $(P<0.001)$ without bag distension as well as with all bag distension, irrespective of the volume used (Fig. 3A). The AP lengths of PRM at rest and contraction were $5.0 \pm 0.3 \mathrm{~cm}$ and $4.6 \pm 0.1 \mathrm{~cm}$, respectively (Fig. 3A). Smaller distensions of vagina with bag did not result in any opening of the vaginal high pressure zone (HPZ) because water in the bag was displaced towards the cranial and caudal ends of bag by vaginal HPZ and there was no effect on the AP length of the PRM. AP length of PRM increased

\section{A}

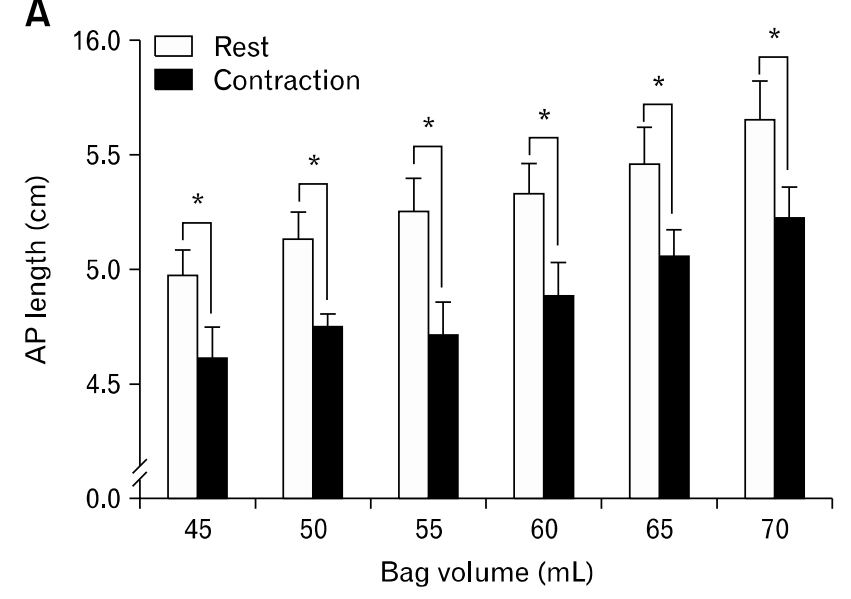

C

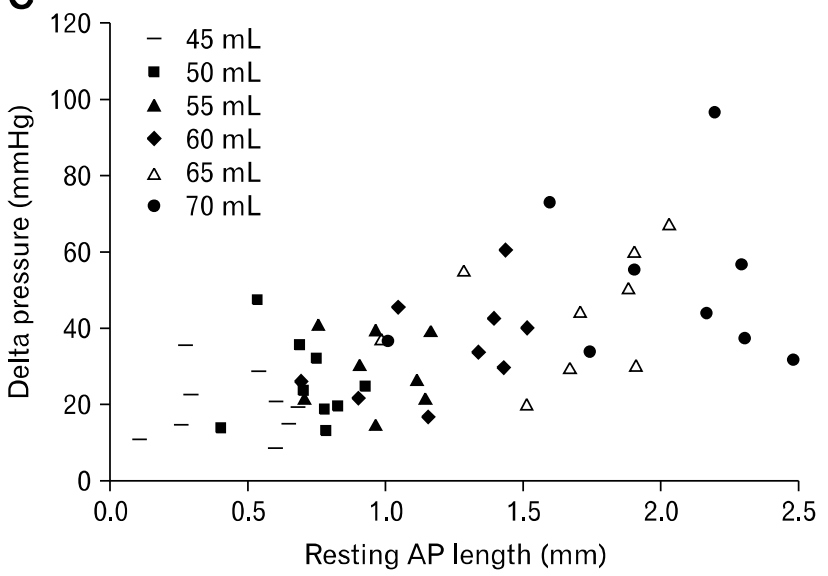

in a linear fashion as balloon volume was increased; correlation coefficients were calculated for each subject and $r$-values ranged from 0.69 to 0.98 (mean, 0.89) at rest and 0.65 to 0.98 (mean, 0.85 ) with contraction. At bag volume of $70 \mathrm{~mL}$, rest and contraction PRM length was $5.7 \pm 0.2$ and $5.2 \pm 0 \mathrm{~cm}$, respectively. Bag pressures at rest and squeeze increased with the increase in bag volume in a linear fashion $(P<0.05)$. At bag volume of 70 $\mathrm{mL}$, bag pressures were $54 \pm 9$ and $105 \pm 11 \mathrm{mmHg}$, at rest and contraction, respectively (Fig. 3B). There was also a direct and linear relationship between delta bag pressure (difference between squeeze and rest) and AP length of PRM before the onset of contraction $(r=0.625 ; P<0.001)$ (Fig. 3C).

\section{Effect of Probe Size on Vaginal Canal Pressure}

Our earlier studies show that there is a high pressure zone in the vagina that extends from the hymen to approximately $3 \mathrm{~cm}$ in the cranial direction. ${ }^{7}$ Cranial to the vaginal high pressure zone, vaginal pressure reflects intra-abdominal pressure. Sleeve sensor

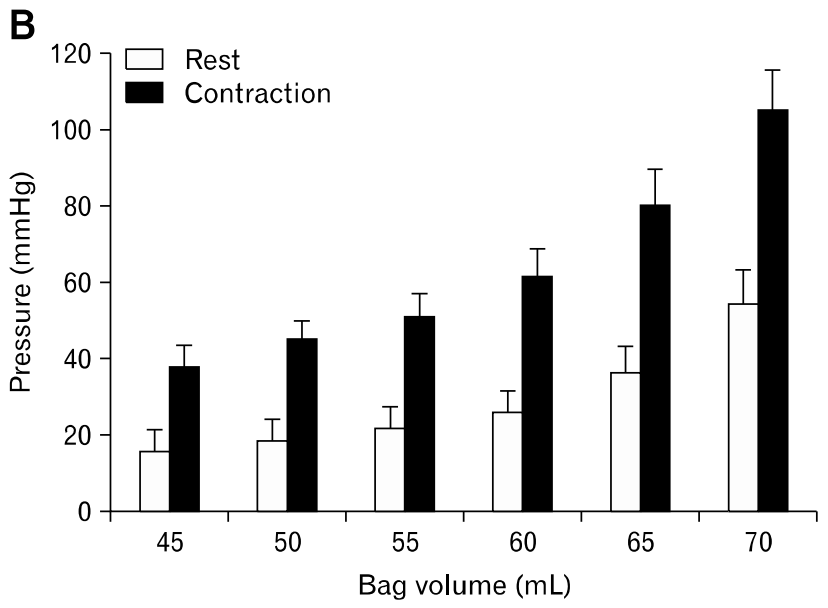

\footnotetext{
Figure 3. Anterior posterior (AP) length changes of puborectalis muscle (PRM) with vaginal distension. (A) AP length of the pelvic floor hiatus, which reflects the PRM length, increases with the increase in bag volume. A significant $\left({ }^{*} P<0.001\right)$ decrease in the PRM length with pelvic floor contraction in the presence of bag. (B) Effect of bag volume on bag pressure. An increase in bag pressure with an increase in bag volume, both at rest as well as with squeeze. At each bag volume the pelvic floor contraction is associated with a significant $(P<0.05)$ increase in bag pressure. (C) Scattered plot showing the relationship between delta pressure (difference between squeeze and rest pressure) and AP length of the pelvic floor hiatus at each bag volume prior to the onset of squeeze $(r=0.625 ; P<0.001)$.
} 

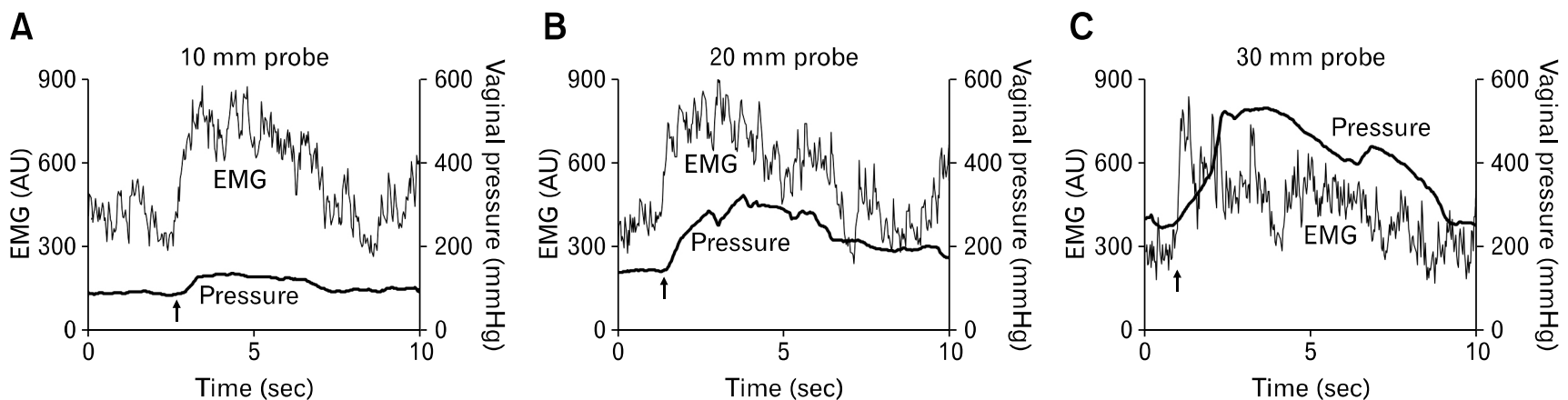

Figure 4. Representative tracing of vaginal pressure and puborectalis muscle electromyogram (EMG) with 3 different-sized vaginal probes. These tracings show that with the increase in probe size, vaginal pressure (both rest and squeeze) increases without any increase in the EMG activity. Arrows indicate onset of squeeze.

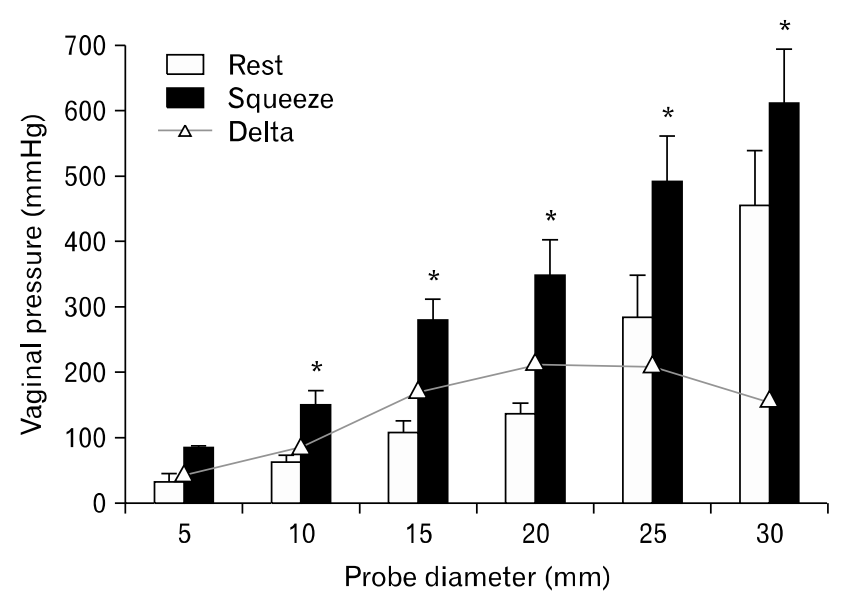

Figure 5. Effect of probe size on vaginal high pressure zone (HPZ) pressure at rest and squeeze. Asterisk $\left(^{*}\right)$ denotes a significant increase $(P<0.05)$ in the rest and squeeze pressures. Vaginal pressure is significantly higher when measured with $10,15,20,25$ and $30 \mathrm{~mm}$ diameter probes as compared to $5 \mathrm{~mm}$ probe.

records the highest pressure along the vaginal $\mathrm{HPZ}$ and side hole above the sleeve records the vaginal pressure above the vaginal HPZ (intra-abdominal pressure). ${ }^{18}$ With voluntary squeeze there is increase in pressure of the vaginal HPZ. Effects of probe size on the vaginal $\mathrm{HPZ}$, at rest and squeeze $(\mathrm{mmHg}$; mean $\pm \mathrm{SEM})$ are shown in Figures 4 and 5. Vaginal pressure with $5 \mathrm{~mm}$ diameter sleeve sensor (without any probe holder) was $30 \pm 9$ and $76 \pm 15$ $\mathrm{mmHg}$, at rest and squeeze respectively $(\mathrm{n}=10)$. Rest and squeeze vaginal $\mathrm{HPZ}$ pressures increased with the increase in probe size $(P$ $<0.05)$. Squeeze pressure with the largest probe size $(30 \mathrm{~mm})$ was $611 \pm 79$ as compared to $76 \pm 15$ with the $5 \mathrm{~mm}$ probe $(P<0.05$; Fig. 5).

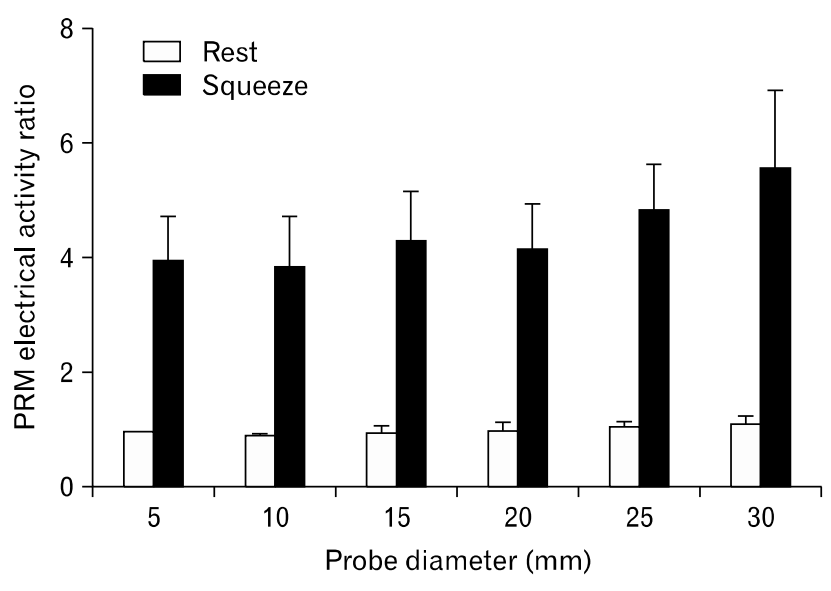

Figure 6. Effect of probe size on puborectalis muscle electromyographic (EMG) activity at rest and squeeze. Note that there is no increase in the EMG activity, neither at rest nor at squeeze, with the increase in probe size.

\section{Effect of Probe Size on the Electromyographic Activity of Puborectalis Muscle}

Tonic EMG was recorded in the PRM at rest suggesting a tonic contraction of PRM in the resting state. With voluntary squeeze, a 3-4 folds increase in the EMG activity was observed (Fig. 4 and 6). With the increase in probe size there was no increase in the rest EMG activity of the PRM. Similarly, the squeeze EMG activity of PRM was not affected by the increase in probe size (Fig. 4 and 6).

\section{Discussion}

Purpose of this study was to determine if, similar to EAS, PRM also operates at a short sarcomere length. Our earlier studies show that the vaginal HPZ is related to the PRM contraction. ${ }^{6,7}$ 
In the present study we demonstrate that distension of vagina increases PRM length, as well as the rest and squeeze vaginal HPZ pressures. Delta or change in the vaginal pressure associated with voluntary contraction increases with the increases in probe size but there is no increase in the EMG/neural drive to PRM. As reasoned in the following paragraphs, these observations support our hypothesis that similar to human EAS; human PRM also operates at the short sarcomere length.

Our studies in rabbit and humans show that the EAS muscle operates at the short sarcomere length, or in other words below the optimal muscle length. ${ }^{13}$ According to the sliding filament theory, optimal muscle length is the one that generates maximal force/tension for the maximal neural input. ${ }^{10}$ In rabbit studies, we used a probe system, similar to the one in the current study and observed that the increase in probe size increases EAS muscle sarcomere length. We measured sarcomere length using laser diffraction method and thin filament (actin) length by "distributed deconvolution and immunohistochemistry images" 19 in rabbit studies. Optimal sarcomere length is equal to $2 \times$ thin filament length plus $\mathrm{Z}$ band width of sarcomere. Based on the above measurements, operational and optimal EAS muscle lengths in rabbit were measured to be approximately 2.1 and $2.6 \mu \mathrm{m}$ respectively. ${ }^{13}$ In other words, EAS muscle length operates at approximately $20 \%$ shorter than its optimal length in vivo. Measurement of sarcomere length by laser diffraction technique and thin filament (actin) length by immunohistochemistry requires excision/harvesting of muscle, which is not practical for the human study.

For the human studies, we measured anal canal pressure with different size probes along with EMG activity of the EAS muscle and found that increase in anal probe diameter results in an increase in the anal canal squeeze pressure and EAS muscle tension, without increase in the EMG activity. Increase in anal canal pressure/EAS muscle tension in the absence of an increase in the neural drive proves that similar to rabbit, human EAS muscle also operates at the sub-optimal or short sarcomere length. Vaginal $\mathrm{HPZ}$ pressure at rest and squeeze is related to the contraction of PRM. ${ }^{6,7}$ Since the original observation by Kegel, several investigators have used various techniques to measure vaginal $\mathrm{HPZ}{ }^{20-24}$ In our laboratory, we have characterized the static and dynamic characteristics of vaginal pressure using several techniques: (1) infusion manometry and rapid pull-through technique, (2) 3D $\mathrm{US}^{6}$ and $\mathrm{MRI}^{7}$ of vaginal constriction caused by vaginal high pressure zone, (3) novel, high definition manometry technique ${ }^{7}$ and (4) in the present study, we used yet another technique, i.e., sleeve sensor, ${ }^{16}$ which measures the highest pressure along the vaginal canal and therefore records peak pressure of the vaginal HPZ. Advantage with the sleeve sensor is that by placing it in different diameter probes one can induce vaginal distension and thus alter PRM length while measuring vaginal HPZ at the same time. We observed that vaginal $\mathrm{HPZ}$ pressure increases in a linear fashion with the increase in probe sizes. Bo et al observed that vaginal $\mathrm{HPZ}$ strength is related to the diameter of the vaginal probe used. ${ }^{20}$ Several investigators have used a "tuning fork type device (dynamometer)" with the ability to expand the distance between the 2 forks to measure the pelvic floor contraction force. $^{25-28}$ Findings from these devices show that contraction force increases in a linear fashion with the increase in the distance between the 2 forks. Change in the distance between the two forks is likely to increase the length of pelvic floor hiatus (PRM), similar to distension of the vagina by a bag in our study. There are 2 possible explanations why vaginal $\mathrm{HPZ}$ pressure may increase with the increase in probe size: (1) an increase in the neural drive to PRM (central mechanism) or (2) an increase in the PRM sarcomere length if PRM were to operate at short sarcomere length (peripheral mechanism). EMG activity of PRM is a surrogate of the neural drive to muscle and we observed that with the increase in probe size there was neither an increase in the EMG activity at rest nor with voluntary contraction. Increase in vaginal probe size should result in an increase in PRM sarcomere length, similar to what we described in our EAS muscle study. ${ }^{13}$ Therefore, based on aforementioned arguments, we propose that probe size dependent increase in the vaginal $\mathrm{HPZ}$ pressure results from the increase in PRM sarcomere length and the latter is only possible if the PRM were to operate in vivo at a short sarcomere length. On the other hand, if the PRM were to operate at optimal or supra-optimal length in vivo; one would expect a decrease in the vaginal $\mathrm{HPZ}$ pressure with the increase in vaginal probe size. ${ }^{10,29}$ We found that vaginal HPZ pressure with $30 \mathrm{~mm}$ diameter probe was larger than with $25 \mathrm{~mm}$ probe, which means that we did not achieve peak force or optimal sarcomere length even with $30 \mathrm{~mm}$ diameter probe. Since insertion of probes larger than $30 \mathrm{~mm}$ was uncomfortable for subjects, we did not use larger size probes.

One may argue that we used pressure and not muscle tension/stress measurement in our study. For our EAS studies, we measured EAS muscle radius and muscle thickness from US images and using Laplace law calculated the EAS muscle stress. PRM being a "U" shaped muscle does not lend itself to calculation of muscle stress using Laplace's law for circular geometry. However, pressure is directly related to muscle stress/tension and is a legitimate surrogate marker of muscle stress/tension and 


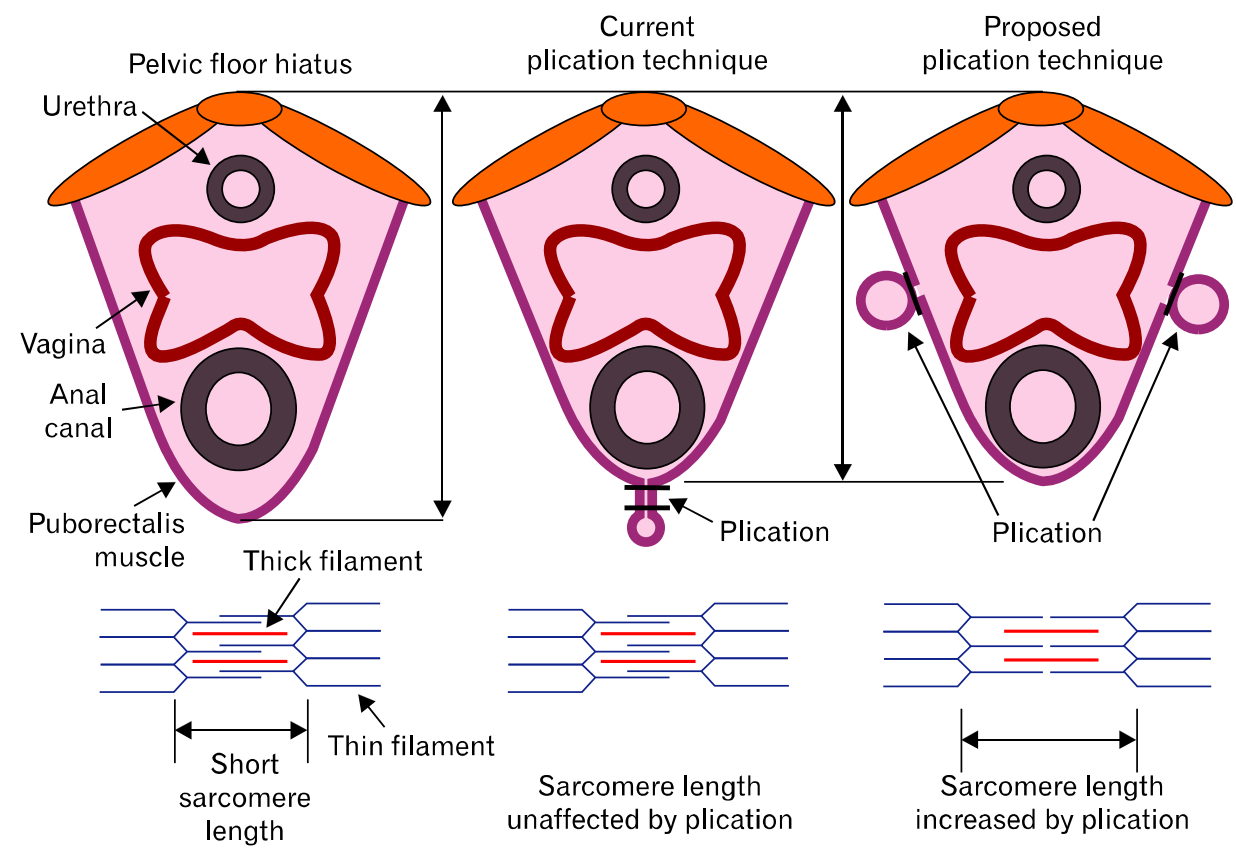

Figure 7. Current and the proposed plication techniques of the pelvic floor hiatus/puborectalis muscle. others have used length-pressure as a surrogate of length-tension measurement. ${ }^{30}$

Our finding that in vivo length of PRM (operation length) is significantly shorter than its optimal length has considerable clinical relevance for the treatment of anal incontinence and possible other pelvic floor muscle disorders (urethral incontinence and vaginal closure function). Our earlier studies show that PRM contraction plays a major role in the anal canal, vagina and urethra closure mechanism. ${ }^{6-8}$ Surgical plication of EAS muscle in rabbits increases its sarcomere length and increases anal canal pressure, in both acute and chronic studies. ${ }^{12,14}$ Surgical plication of the pelvic floor hiatus has been used in the past to treat anal incontinence with mixed results. ${ }^{31}$ However, plication technique used in these studies consisted of closure of hiatus posterior to the anal canal (Fig. 7; mid panel). We propose that adjusting the PRM length by surgical plication of the 2 arms of " $U$ " shaped PRM (Fig. 7; right panel) may be a better strategy because it is more likely to stretch the PRM muscle and increase its sarcomere length given that the fibers of PRM are directed in the anterior-posterior direction. Further studies are needed to determine if the proposed PRM plication is effective in the treatment of patients with anal incontinence, vaginal prolapse and urinary incontinence.

\section{Acknowledgement}

Authors thank Ms Melissa Ledgerwood for her help with manuscript figure.

\section{References}

1. Rao SS. Pathophysiology of adult fecal incontinence. Gastroenterology 2004;126(suppl 1):S14-S22.

2. Culver PJ, Rattan S. Genesis of anal canal pressures in the opossum. Am J Physiol 1986;251(6 Pt 1):G765-G771.

3. Diamant NE, Kamm MA, Wald A, Whitehead WE. AGA technical review on anorectal testing techniques. Gastroenterology 1999;116: 735-760.

4. Padda BS, Jung SA, Pretorius D, Nager CW, Den-Boer D, Mittal RK. Effects of pelvic floor muscle contraction on anal canal pressure. Am J Physiol Gastrointest Liver Physiol 2007;292:G565-G571.

5. Liu J, Guaderrama N, Nager CW, Pretorius DH, Master S, Mittal RK. Functional correlates of anal canal anatomy: puborectalis muscle and anal canal pressure. Am J Gastroenterol 2006;101:1092-1097.

6. Jung SA, Pretorius DH, Padda BS, et al. Vaginal high-pressure zone assessed by dynamic 3-dimensional ultrasound images of the pelvic floor. Am J Obstet Gynecol 2007;197:52, e1-e7.

7. Raizada V, Bhargava V, Jung SA, et al. Dynamic assessment of the vaginal high-pressure zone using high-definition manometery, 3-dimensional ultrasound, and magnetic resonance imaging of the pelvic floor muscles. Am J Obstet Gynecol 2011;203:172, e1-e8.

8. Rajasekaran MR, Sohn D, Salehi M, Bhargava V, Fritsch H, Mittal RK. Role of puborectalis muscle in the genesis of urethral pressure. J Urol 2012;188:1382-1388. 
9. Guaderrama NM, Liu J, Nager CW, et al. Evidence for the innervation of pelvic floor muscles by the pudendal nerve. Obstet Gynecol 2005;106:774-781.

10. Gordon AM, Huxley AF, Julian FJ. The variation in isometric tension with sarcomere length in vertebrate muscle fibres. J Physiol 1966;184:170-192.

11. Mittal RK, Sheean G, Padda BS, Lieber R, Rajasekaran MR. The external anal sphincter operates at short sarcomere length in humans. Neurogastroenterol Motil 2011;23:643-e258.

12. Rajasekaran MR, Jiang Y, Bhargava V, Lieber RL, Mittal RK. Novel applications of external anal sphincter muscle sarcomere length to enhance the anal canal function. Neurogastroenterol Motil 2011; 23:70-75, e7.

13. Rajasekaran MR, Jiang $Y$, Bhargava $V$, et al. Length-tension relationship of the external anal sphincter muscle: implications for the anal canal function. Am J Physiol Gastrointest Liver Physiol 2008; 295:G367-G373.

14. Rajasekaran MR, Jiang Y, Bhargava V, Ramamoorthy S, Lieber RL, Mittal RK. Sustained improvement in the anal sphincter function following surgical plication of rabbit external anal sphincter muscle. Dis Colon Rectum 2011;54:1373-1380.

15. Sivri B, Mittal RK. Reverse-perfused sleeve: an improved device for measurement of sphincteric function of the crural diaphragm. Gastroenterology 1991;101:962-969.

16. Dent J. A new technique for continuous sphincter pressure measurement. Gastroenterology 1976;71:263-267.

17. Mittal RK, Rochester DF, McCallum RW. Electrical and mechanical activity in the human lower esophageal sphincter during diaphragmatic contraction. J Clin Invest 1988;81:1182-1189.

18. Linehan JH, Dent J, Dodds WJ, Hogan WJ. Sleeve device functions as a Starling resistor to record sphincter pressure. Am J Physiol 1985; 248(2 Pt 1):G251-G255.

19. Littlefield R, Fowler VM. Measurement of thin filament lengths by distributed deconvolution analysis of fluorescence images. Biophys J 2002;82:2548-2564.

20. Bo K, Sherburn M. Evaluation of female pelvic-floor muscle function and strength. Phys Ther 2005;85:269-282.

21. Kegel AH. Progressive resistance exercise in the functional restoration of the perineal muscles. Am J Obstet Gynecol 1948;56:238-248.

22. Kegel AH. Physiologic therapy for urinary stress incontinence. J Am Med Assoc 1951;146:915-917.

23. Levitt EE, Konovsky M, Freese MP, Thompson JF. Intravaginal pressure assessed by the Kegel perineometer. Arch Sex Behav 1979; 8:425-430.

24. Logan TG. The vaginal clasp. A method of comparing contractions across subjects. J Sex Res 1975;11:353-358.

25. Dumoulin C, Gravel D, Bourbonnais D, Lemieux MC, Morin M. Reliability of dynamometric measurements of the pelvic floor musculature. Neurourol Urodyn 2004;23:134-142.

26. Dumoulin C, Bourbonnais D, Lemieux MC. Development of a dynamometer for measuring the isometric force of the pelvic floor musculature. Neurourol Urodyn 2003;22:648-653.

27. Miller JM, Ashton-Miller JA, Perruchini D, DeLancey JO. Test-retest reliability of an instrumented speculum for measuring vaginal closure force. Neurourol Urodyn 2007;26:858-863.

28. Constantinou CE, Omata S. Direction sensitive sensor probe for the evaluation of voluntary and reflex pelvic floor contractions. Neurourol Urodyn 2007;26:386-391.

29. Huxley AF, Simmons RM. Proposed mechanism of force generation in striated muscle. Nature 1971;233:533-538.

30. Biancani P, Zabinski MP, Behar J. Pressure tension, and force of closure of the human lower esophageal sphincter and esophagus. J Clin Invest 1975;56:476-483.

31. Matsuoka H, Mavrantonis C, Wexner SD, Oliveira L, Gilliland R, Pikarsky A. Postanal repair for fecal incontinence-is it worthwhile? Dis Colon Rectum 2000;43:1561-1567. 